\section{A dentist's guide to using the BNF: part I}

\author{
D. Wray ${ }^{1}$ and S. M. S. Wagle ${ }^{2}$
}
IN BRIEF
- Explains how to find significant changes in a new edition of the BNF
- Highlights important new information for dentists in BNF 55 (March 2008).
- Explains how to find dental information in the print and digital versions of the BNF.
- Provides details of an e-newsletter service launched recently for dentists and other healthcare professionals.

It was almost four years ago that information about medicines for oral conditions was integrated into the British National Formulary (BNF) proper. The intention was to prepare dentists to prescribe on terms similar to those of their medical colleagues and to make the information on dental and oral conditions accessible to all healthcare professionals who use the $B N F$. In the first of a two-part series, we describe how dentists can find significant changes in a new edition of the BNFand navigate to the dental information in it.

\section{FINDING SIGNIFICANT CHANGES IN A NEW EDITION}

As soon as a new edition of the $B N F$ is published (in March and September each year) you should identify the changes in its content that are relevant to your practice. The publication includes a number of aids to help you do this. The most up-to-date BNF can also be found at http://bnf.org. Registration and use of this website is free for all UK dentists.

\section{Insert}

The print version includes an Insert that summarises the background to several key changes (Fig. 1). A copy of the Insert can also be found at http://bnf. org by going to 'Updates' and clicking on 'What's new in BNF?' (Fig. 2).

While reading the Insert for BNF 55 (March 2008) ${ }^{1}$ you will find that:

- Antimicrobial prophylaxis is no longer recommended for the prevention of endocarditis in patients who are undergoing dental procedures

Chairman of BNF Dental Advisory Group/Dean of the Dental School, Glasgow Dental Hospital and School, 378 Sauchiehall Street, Glasgow, G2 3JZ; ${ }^{2 *}$ Assistant Editor, BNF Publications, Royal Pharmaceutical Society of Great Britain, 1 Lambeth High Street, London, SE1 7JN

${ }^{*}$ Correspondence to: Shama Wagle

shama.wagle@rpsgb.org

Refereed Paper

DOI: $10.1038 /$ sj.bdj.2008.294

${ }^{\circledR}$ British Dental Journal 2008; 204: 437-439
- An anticoagulated patient can undergo an extraction if his INR is less than 4 before the procedure

- The paediatric dose of adrenaline for anaphylaxis has been revised to reflect the new guidelines from the UK Resuscitation Council

- Details are provided of how the BNF is constructed.

\section{Changes for this edition}

This section is located near the beginning of the print version (pp xi-xiii of $B N F$ 55, March 2008) (Fig. 3). For ease of identification, the margins of these pages are marked in blue stripes. The section provides a list of significant changes, dose changes, classification changes, new names and new preparations that have been incorporated into a new edition, as well as a list of preparations that have been discontinued since the last edition. A quick glance at this section shows that the legal status of midazolam has changed from a schedule 4 to a schedule 3 controlled drug. The changes section can also be found by logging on to the BNF content at http:// bnf.org and going to 'General Information and Late Additions'.

\section{Changes to the Dental Practitioners' Formulary}

The 'Dental Practitioners' Formulary' can be found near the back of the print version but its exact location can be found

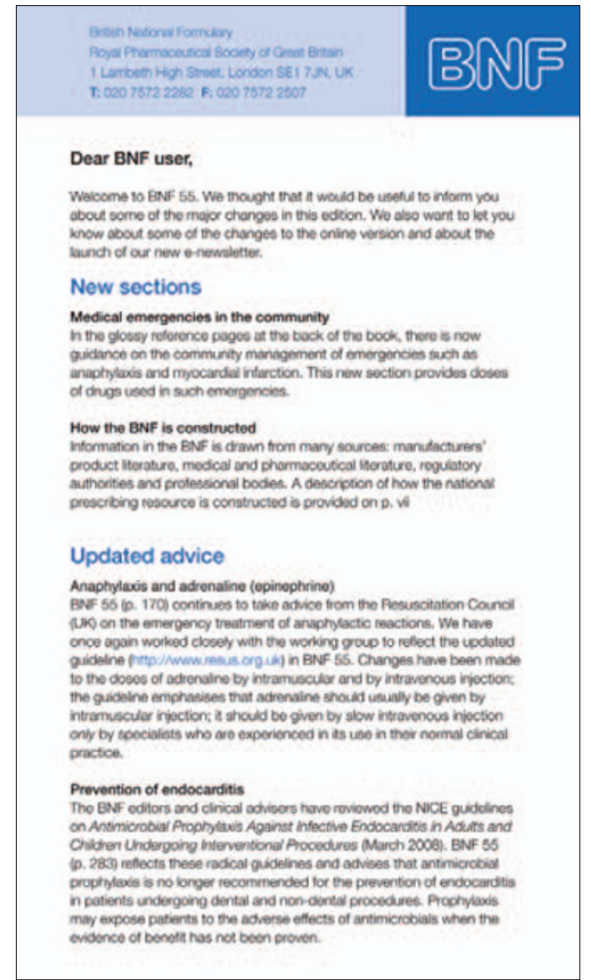

Fig. 1 The $B N F$ Insert summarises the background to several key changes in a new edition of the $B N F$

using the Contents page (or the hierarchy in the digital version). Changes to the formulary are found at the end of the Dental List. With the agreement of government dental officers, a number of new additions have been made to the List in BNF 55 (Fig. 4).

\section{Changes to the Appendices}

Drug entries that have been amended 
or introduced in Appendices 1-5 (Drug Interactions, Liver Disease, Renal Impairment, Pregnancy, Breast-feeding) are underlined in the print version.

\section{E-newsletter}

In March 2008, an e-newsletter service will be launched to alert dentists and other healthcare professionals to:

- Details of significant updates in the BNF

- Tips for using the BNF and BNF for Children (BNFC) effectively

- Examples of prescribing excellence and case studies

- Details of developments on BNF publications.

So many changes are made to each new edition of the BNF that not all of them can be accommodated in the Insert and the Changes section. We encourage you to review regularly the prescribing information on drugs that you prescribe frequently.

\section{FINDING DENTAL INFORMATION IN THE BNF}

Although the dental information is scattered throughout the $B N F$, extra aids have been added to help you access this information.

\section{Prescribing in dental practice}

'Prescribing in Dental Practice' is located near the front of the BNF (pp 21, BNF 55, March 2008). On its very first page, you will find a contents list dedicated to drugs and topics of relevance to dentists, together with cross references to the prescribing notes in the appropriate sections of the BNF (Fig. 5). For example, a review of this list shows that information on the local treatment of oral infections is located in chapter 12 (Ear, Nose and Throat) while information on the systemic treatment of these infections is found in chapter 5 (Infections).

Advice on the management of medical emergencies is located within 'Prescribing in Dental Practice'. In BNF 55, chorphenamine, hydrocortisone and salbutamol injections have been removed from the emergency drug list for dental practice because these are not considered to be first-line drugs for use in this setting. Advice on the management

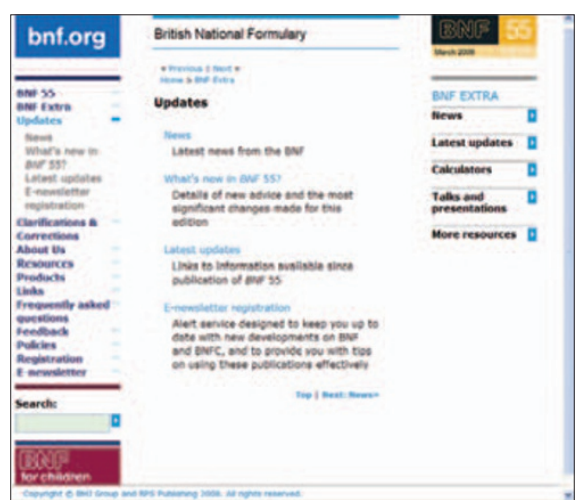

Fig. 2 A copy of the BNF Insert can be found by going to 'Updates' and clicking on 'What's new in BNF?'

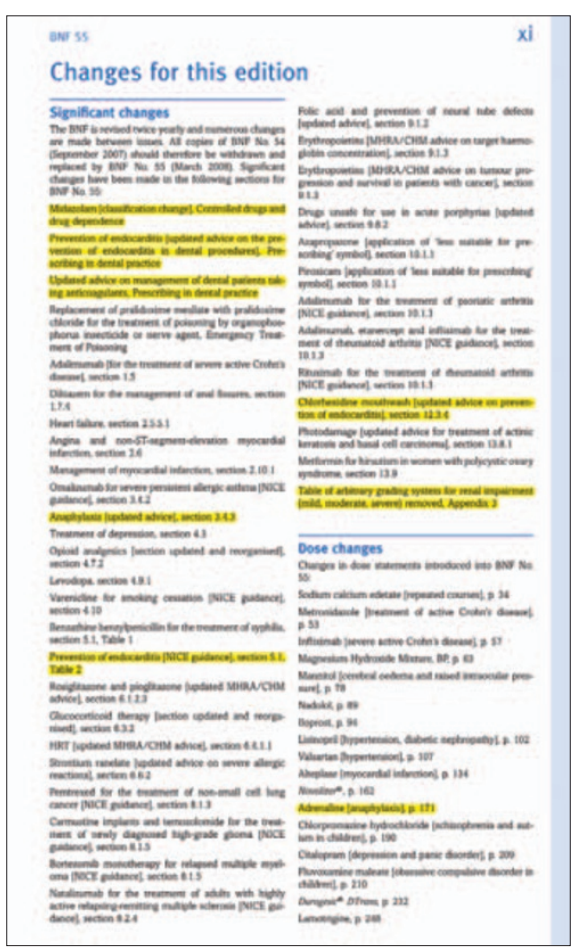

Fig. 3 'Changes for this Edition' provides a list of significant changes, dose changes, classification changes, new names, new preparations and discontinued preparations in a new edition. For ease of identification, the margins of these pages in the print version of the $B N F$ are marked in blue. You can find further details of a change by going to the cross-reference provided (in the digital version you can do this at the click of a hyperlink)

of medical problems in dental practice is also located within this section. Here you will find further details of the new guidance on the prevention of endocarditis and advice on the management of anticoagulated patients undergoing dental surgery (see Example 1).

\section{Index}

Entries are included in alphabetical order of drugs and conditions in the

\section{Sodium Fluoride Toothpaste $1.1 \%$ PoM (proprietary product: Duraphat '5000 ppm' Toothpaste). sodium fluoride $1.1 \%$ \\ Changes to Dental Practitioners' Formulary since September 2007 Additions \\ Aciclovir Tablets BP, $800 \mathrm{mg}$ \\ Beclometasone Dipropionate Aerosol Inhalation 50 micrograms/metered dose, DPF \\ Betamethasone Soluble Tablets, 500 micrograms, DPF Diclofenac Sodium Tablets, BP \\ Lidocaine Spray $10 \%$, DPF \\ Miconazole Cream, BP \\ Saliva Stimulating Tablets, DPF

Fig. 4 Changes to the 'Dental Practitioners' Formulary' are included at the end of the 'Dental List'

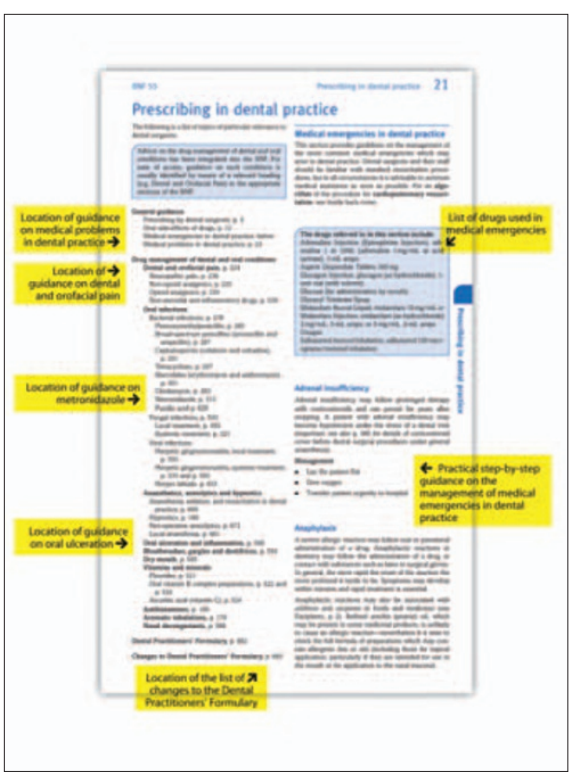

Fig. 5 'Prescribing in Dental Practice' includes a contents list that is the gateway to the information on drugs and topics of relevance to dentists in the $B N F$. Advice on the management of medical emergencies and medical problems in dental practice is also located in this section

Index and can be used to access dental information. There is also a specific entry for 'Dental Prescribing' which brings together various topics of relevance to dentists. In the digital BNF you can use the Google search engine to browse for a drug or condition; the search results are displayed in order of relevance.

\section{Side-headings}

In the prescribing notes, numerous 
short half-life of pethidine necessitates frequent injections.

Dental and orofacial pain Analgesics should be used judiciously in dental care as a temporary measure until the cause of the pain has been dealt with.

Dental pain of inflammatory origin, such as that associated with pulpitis, apical infection, localised osteitis (dry socket) or pericoronitis is usually best managed by treating the infection, providing drainage, restorative procedures, and other local measures. Analgesics provide temporary relief of pain (usually for about 1 to 7 days) until the causative factors have been brought under control. In the case of pulpitis, intra-osseous infection or abscess, reliance on analgesics alone is usually inappropriate.

Similarly the pain and discomfort associated with acute problems of the oral mucosa (e.g. acute herpetic gingivostomatitis, erythema multiforme) may be relieved by benzydamine mouthwash or spray (see p. 591) until the cause of the mucosal disorder has been dealt with However, where a patient is febrile, the antipyretic action of paracetamol (see p. 226) or ibuprofen (see p. 540) is often helpful.

The choice of an analgesic for dental purposes should be based on its suitability for the patient. Most dental pain is relieved effectively by non-steroidal anti-inflammatory drugs (NSAIDs). NSAIDs that are used for dental pain include ibuprofen, diclofenac, and aspirin; for further details see section 4.7.1 and section 10.1.1 Paracetamol has analgesic and antipyretic effects but no anti-inflammatory effect.

Opioid analgesics (section 4.7.2) such as dihydrocodeine and pethidine act on the central nervous system and are traditionally used for moderate to severe pain However, opioid analgesics are relatively ineffective in dental pain and their side-effects can be unpleasant.

Fig. 6 In the prescribing notes, sideheadings facilitate the identification of advice on oral conditions

\footnotetext{
Diclofenac Sodium (Non-proprietary) $\mathrm{Pol}$

Tablets, e/c, diclofenac sodium $25 \mathrm{mg}$, net price 84 tab pack $=£ 1.20 ; 50 \mathrm{mg}, 84$-tab pack $=£ 2.03$. Label: 5 , 25

Note Other brands include Defenac ${ }^{\oplus}$, Dicloflex $x^{\oplus}$, Diclozip ${ }^{\oplus}$, Fenacto $^{\oplus}$, Flamrase ${ }^{\circledR}$

Dental prescribing on NHS Diclofenac Sodium Tablets may be prescribed

Suppositories, diclofenac sodium $100 \mathrm{mg}$, net price $10=£ 3.04$

Brands include Econac

Injection, diclofenac sodium $25 \mathrm{mg} / \mathrm{mL}$. Net price 3$\mathrm{mL}$ amp $=83 \mathrm{p}$
}

Note Licensed for intramuscular use

Fig. 7 Preparations that you can prescribe using NHS form FP10D (GP14 in Scotland, WP10D in Wales) can be identified by means of a note headed 'Dental prescribing on the NHS' side-headings facilitate the identification of advice on oral conditions (Fig. 6).

\section{Dental prescribing on the NHS}

In the body of the $B N F$, preparations that you can prescribe using NHS form FP10D (GP14 in Scotland, WP10D in Wales) can be identified by means of a note headed 'Dental prescribing on the NHS' (Fig. 7).

\section{Example 1}

You need to conduct an extraction in a woman with a mechanical aortic valve who takes warfarin. Her Anticoagulation Record shows that her INR is monitored weekly, her last INR taken four days ago was 3.9, and that she has had several INR measurements greater than 4.5 in the last two months.

1. When should you measure this patient's INR in relation to the dental procedure?

2. At what INR can you proceed with the extraction?

3. Should you prescribe antimicrobial prophylaxis against infective endocarditis for this patient?

The section on 'Thromboembolism' in 'Medical Problems in Dental Practice' (BNF55) indicates that this patient has an unstable INR because she requires weekly monitoring of her INR and has some INR measurements greater than 4 in the last two months. As the INR is unstable, you should review the case with the clinician responsible for her anticoagulation. Her INR should be assessed within 24 hours of the dental procedure. If this INR is below 4, then the patient can continue warfarin without dose adjustment, and the extraction can be conducted while taking measures to minimise bleeding.

The section on 'Infective Endocarditis' in 'Medical Problems in Dental Practice' (BNF55) shows that antibacterial prophylaxis is not recommended for the prevention of endocarditis in this patient. Such prophylaxis may expose her to the adverse effects of antibacterials when the evidence of benefit has not been proven. Routine daily activities such as tooth brushing may present a greater risk of bacteraemia than a single dental procedure.

She should be advised to maintain good oral hygiene and told to report any unexplained illness that develops after dental treatment. Any infection should be treated promptly and treated appropriately to reduce the risk of endocarditis.

\section{FINAL REMARKS}

We encourage you to spend some time getting to know your BNF - you will soon find that it becomes an indispensable prescribing companion. Furthermore, by keeping up-to-date with the changes in each new edition of the $B N F$, you can ensure that your patients benefit from prescribing decisions based on the latest evidence.

We thank L. Oliver, P. Wilder and P. Lee for preparing images of the $B N F$.

1. Joint Formulary Committee. British National Formulary. 55th ed. London: British Medical Association and Royal Pharmaceutical Society of Great Britain, 2008. 